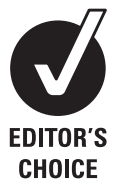

See Editorial, p 2

- Additional data are published online only. To view these files please visit the journal online (http://sti.bmi.com/content/88/ 1.toc).

For numbered affiliations see end of article.

\section{Correspondence to}

Dr Claudia Estcourt, Centre for Infectious Disease: Sexual Health \& HIV, Blizard Institute of Cell and Molecular Science, Barts \& The London School of Medicine \& Dentistry, Barts and the London NHS Trust, Barts Sexual Health Centre, Kenton \& Lucas Wing, St Bartholomew's Hospital, West Smithfield, London EC1A 5BE, UK; c.s.estcourt@qmul.ac.uk

Accepted 23 June 2011 Published Online First 27 July 2011

\title{
Can we improve partner notification rates through expedited partner therapy in the UK? Findings from an exploratory trial of Accelerated Partner Therapy (APT)
}

\author{
Claudia Estcourt, ${ }_{1}^{1}$ Lorna Sutcliffe, ${ }^{2}$ Jackie Cassell, ${ }^{3}$ Catherine H Mercer, ${ }^{4}$ \\ Andrew Copas, ${ }^{4}$ Laura James, ${ }^{4}$ Nicola Low, ${ }^{5}$ Patrick Horner, ${ }^{6}$ Michael Clarke, \\ Merle Symonds, ${ }^{8}$ Tracy Roberts, ${ }^{9}$ Angelos Tsourapas, ${ }^{9}$ Anne M Johnson ${ }^{4}$
}

\begin{abstract}
Objectives To develop two new models of expedited partner therapy for the UK, and evaluate them for feasibility, acceptability and preliminary outcome estimates to inform the design of a randomised controlled trial (RCT).
\end{abstract}

Methods Two models of expedited partner therapy (APTHotline and APTPharmacy), known as 'Accelerated Partner Therapy' (APT) were developed. A nonrandomised comparative study was conducted of the two APT models and routine partner notification (PN), in which the index patient chose the PN option for his/her partner(s) in two contrasting clinics.

Results The proportion of contactable partners treated when routine PN was chosen was 42/117 (36\%) and was significantly higher if either APT option was chosen: APTHotline 80/135 (59\%), $p=0.003$; APTPharmacy $29 / 44(66 \%) p=0.001$. However, partner treatment was often achieved through other routes. Although 40-60\% of partners in APT groups returned urine samples for sexually transmitted infection (STI) testing, almost none accessed HIV and syphilis testing. APT options appear to facilitate faster treatment of sex partners than routine PN. Preferences and recruitment rates varied between sites, related to staff satisfaction with existing routine PN; approach to consent; and possibly, characteristics of local populations.

Conclusions Both methods of APT were feasible and acceptable to many patients and led to higher rates of partner treatment than routine PN. Preferences and recruitment rates varied greatly between settings, suggesting that organisational and cultural factors may have an important impact on the feasibility of an RCT and on outcomes. Mindful of these factors, it is proposed that APT should now be evaluated in a cluster RCT.

\section{INTRODUCTION}

Partner notification (PN) aims to control the spread of sexually transmitted infections (STIs) by reducing the duration of infectivity, ${ }^{12}$ and involves informing the infected person's sexual partners of the exposure, offering diagnosis and treatment and providing advice about prevention. ${ }^{3}$

Although the need for PN is generally accepted, it is unclear which approaches work best. PN in the UK generally takes place through 'patient referral', in which a person with an STI refers their own partner(s) to services for diagnosis and treatment.
Patients can be supported with advice, leaflets or counselling. Outside the UK, various forms of expedited partner therapy are sometimes used. The most common method is patient-delivered partner therapy, ${ }^{4}$ which involves treating the sex partners of patients with chlamydia or gonorrhoea by providing prescriptions or drugs to the patient without a healthcare provider first examining the partner. ${ }^{5}$ Patient-delivered partner therapy results in fewer re-infections than patient referral for some STIs. ${ }^{6}$ However, it does not include a medical assessment of the sex partner and so fails to comply with current UK prescribing guidance. ${ }^{7}$

Here we aimed to assess the potential for expedited partner therapy to contribute to STI control in the UK. We developed 'Accelerated Partner Therapy (APT)', which complies with UK prescribing regulations. We define APT as PN strategies that reduce the time for sex partners to be treated and include remote or face-to-face sex partner assessment by an appropriately qualified healthcare professional. Specific objectives were to develop two new models of APT; to determine the acceptability and feasibility of APT in sexual health clinics; to obtain preliminary evidence of efficacy of APT compared with routine $\mathrm{PN}$ in preparation for a randomised controlled trial; to obtain cost data for the APT strategies for a preliminary economic evaluation, described elsewhere.

\section{METHODS}

\section{Study design}

The design comprised a non-randomised comparative study of two APT models and routine PN, in which the index patient chose the PN option for his/her partner(s).

Using established guidance for development and evaluation of complex interventions, ${ }^{8} 9$ we developed two APT models using published evidence on characteristics of expedited partner therapy evaluated elsewhere, ${ }^{6} 10^{11}$ taking into account UK prescribing guidance. ${ }^{7}$ We refined the models in an iterative, qualitative process, involving an exploration of the acceptability and feasibility of the APT models in a study of sexual health clinic attenders, ${ }^{12}$ and focus group discussions with healthcare professionals (one for each site, with health advisers, clinic nurses, community pharmacists and consultant genitourinary medicine (GUM) doctors). 
An electronic PN database was developed based on CDC standards for surveillance, ${ }^{13}$ and current PN practice. This was implemented in both clinics 3 months before the trial to determine baseline PN outcomes. Ethical approval was obtained (Norfolk Research Ethics Committee, REC:06/O0101/3).

The trial took place in two contrasting sexual health clinics: clinic A, central London (high proportion of commuters); and clinic B, non-London urban population and the sole GUM service in the area, with a high proportion of STI diagnoses made in primary care. ${ }^{14}$ Pre-trial PN in both clinics was mostly by patient referral but practices varied. In clinic A, a health adviser or nurse practitioner provided advice on $\mathrm{PN}$ and supplied contact slips, clinic opening times and infection-specific information for partners. There was readiness to change and improve $\mathrm{PN}$ within the clinic. In clinic B, a health adviser provided advice on $\mathrm{PN}$ and supplied a standard letter including possible treatment regimens, for the sex partner to take to a health provider. This was popular with staff and perceived to be liked by patients.

Index patients were $\geq 16$ years with a laboratory diagnosis of: Chlamydia trachomatis and/or Neisseria gonorrhoeae (men and women) or non-gonococcal urethritis ((NGU) men only), and at least one contactable sex partner (a partner within the UK whom the index patient could contact by telephone). Exclusion criteria for index patients included inability to understand and read English and give consent; or coexistent infection with other STIs-for example, Treponema pallidum and/or HIV. Exclusion criteria for sex partners were age $<16$ years; pregnancy; symptoms of complicated infection; contraindications to azithromycin and/or cefixime; inability to understand and read English and give consent.

Trained health advisers and senior nurses identified index patients at the PN interview and sought consent to participate. They offered participants, for each contactable sex partner, a choice between three PN methods. The practitioner entered details on the APT database and generated a unique relationship APT Study number and the standard code for the index infection(s). The index patient was instructed to give this number (and the study web address and paper copies of the study information leaflets) to their partner, in addition to other routine elements of the PN interview.

When the sex partner engaged with the allocated PN method, a trained healthcare professional explained the study and sought consent from the partner to participate. Figure 1 describes the APT interventions in each clinic.

\section{APTHotline}

A health adviser or senior nurse assessed the partner by telephone using a standard consultation guide. In clinic A, the partner could collect the treatment pack from the clinic reception or have it delivered by the index patient. Clinic B operated an additional step owing to differences in staffing mix, in which the doctor also needed to assess the partner by telephone before collection of the treatment pack from the clinic reception or delivery by the index patient.

\section{APTPharmacy}

The sex partner attended a participating pharmacy for a consultation with a trained community pharmacist who supplied the treatment pack. Each clinic was linked to three community pharmacies, in which one or two pharmacists had undertaken $3 \mathrm{~h}$ training in the clinical management of partners of people with chlamydia, gonorrhoea or NGU by Patient Group Directive (PGD) for azithromycin and cefixime. Both APT options included an 'assertive invitation' to the partner to attend a local sexual health clinic for a fast-track HIV and syphilis test.

'Routine PN' was each clinic's pre-trial standard PN practice (figure 1).
Figure 1 Accelerated Partner Therapy (APT) clinical management pathways. ${ }^{*}$ APT PIN: personal identification number, which was a unique study number for each index patient. (A) In clinic $A$ the health advisers were nursequalified which meant that they could provide treatment under Patient Group Directive (PGD) without needing a doctor to authorise the medication. (B) In clinic B few health advisers were nurse-qualified. This meant that a doctor needed to authorise treatment by speaking to the sex partner in order to satisfy prescribing guidance. (C) APT treatment packs included: azithromycin $1 \mathrm{~g}$ in a blister pack or cefixime $400 \mathrm{mg}$ in a plastic bottle (partners of indexes with $N$ gonorrhoeae and partners of coinfected indexes received both antibiotics); relevant drug information sheets which included contraindications, drug interactions and possible side effects: a urine nucleic acid amplification test sample collection kit for $C$ trachomatis and $N$ gonorrhoeae and appropriate packaging so that the contact could mail it back to the study laboratory; condoms, full details of the study. Clinic A routine partner notification (PN): interview with health adviser or nurse practitioner, provision of contact slip, infection-specific information and advice for partner to attend clinic/GP for treatment. Clinic B routine PN: interview with health adviser, provision of clinic's own letter for sex partner to take to health provider in which possible treatment regimens are described, contact slip, infectionspecific information and advice for partner to attend clinic/GP for treatment.

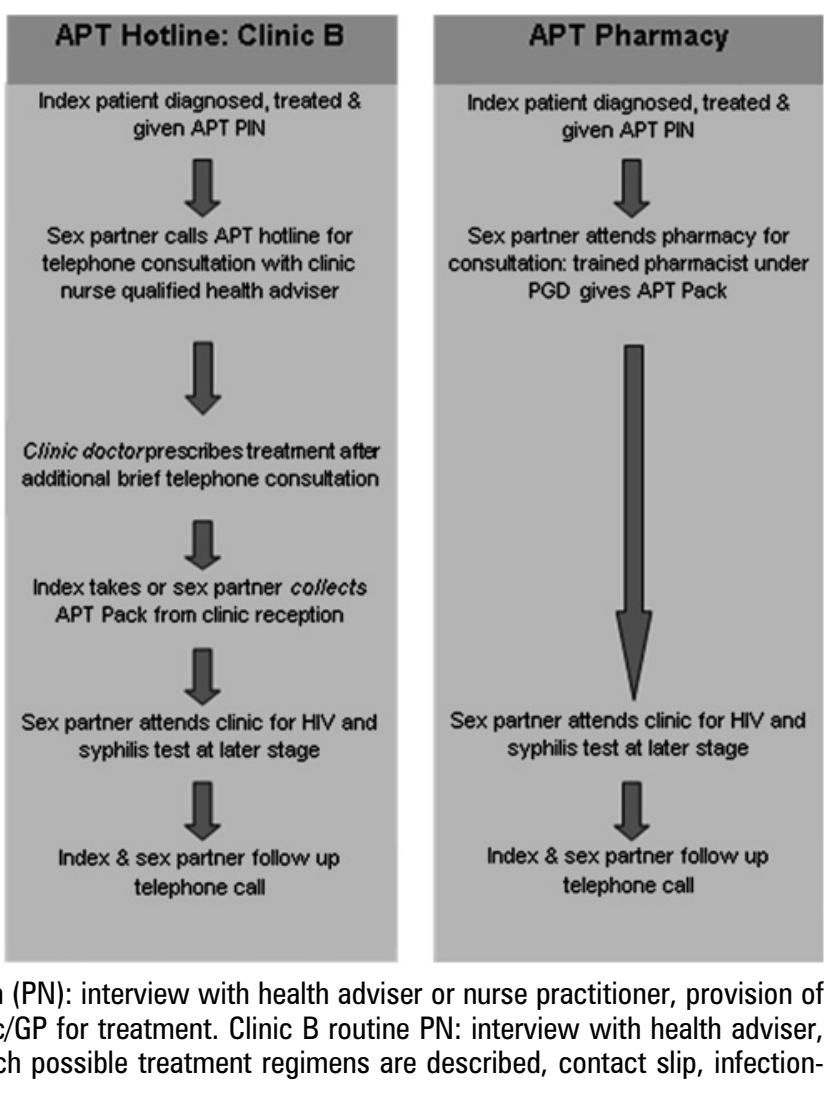




\section{Evaluation framework}

The primary outcome was the proportion of sex partners for each PN intervention assumed treated 4-6 weeks after index patient diagnosis. We ascertained this in three ways. First, we sought evidence of the sex partner engaging with the allocated PN method (attendance at clinic, pharmacy or telephone call to hotline). If this was not recorded then we used information from the sex partner telephone follow-up interview, but if this interview was not obtained then we used information from the index telephone follow-up interview. Secondary outcomes included proportion of partners tested for $N$ gonorrhoeae, $C$ trachomatis, syphilis and HIV; factors associated with index choice of PN option; time taken to achieve partner treatment.

We assessed time to treatment only for partners documented as treated according to the method selected by the index patient. The date treated was taken as follows: APTPharmacy-date that the partner attended the pharmacy; routine $\mathrm{PN}$-date that the partner attended the clinic; APTHotline-date that the partner collected the treatment pack from clinic or the date that the index patient reported giving the pack to the partner.

The power of the study depended on how frequently each of the three possible PN methods was selected by index patients, as well as the likely differences in the rate of the primary outcome. We considered the primary outcome here to relate initially to the index patient (whether one or more contacts were treated). We used a $2 \%$ significance level as three methods were compared through pair-wise comparisons. When comparing the least popular method (assume selected by $20 \%$ ) with a more popular (assume $40 \%$ ) then if the least popular has a $50 \%$ rate of the primary outcome, then a total sample size of 200 index patients provided $80 \%$ power to detect the difference as significant if the underlying outcome rate in the more popular method was $80 \%$. The size also provided $80 \%$ power to detect as significant an underlying difference between two methods of $50 \%$ compared with $75 \%$ if both were selected by $40 \%$ of participants. The primary outcome used in analysis (proportion of contacts treated) had somewhat greater power as some index patients reported more than one contactable partner.

$\chi^{2}$ Tests were used to identify factors associated with choice of PN method and to assess associations for partners between the allocated PN method and our primary outcomes. For this analysis at the 'partner level' the clustering of partners by index was acknowledged through robust SEs. The Mann-Whitney test was used to assess whether times to treatment differed between study arms. Our focus was on testing each APT intervention arm relative to routine $\mathrm{PN}$ and these two $\mathrm{p}$ values are reported. As a sensitivity analysis, the above testing to compare PN methods was repeated for clinic A only, as in clinic $B$ most partners were allocated to one method. All quantitative data manipulation and analyses were conducted using STATA version 8.2 (Stata Corporation, College Station, Austin, Texas, USA).

Process evaluation is summarised in table 1.

\section{RESULTS}

Before the trial in clinic A, 131 index patients with chlamydia, gonorrhoea or NGU reported 174 partners of whom 60 (34\%) were assumed treated (either the partner attended the service or the index patient reported that the partner had received treatment), and in clinic B 128 index patients reported 186 partners of whom 57 (31\%) were assumed treated, consistent with national data. ${ }^{15}$

Figure 2 and table 2 describe the flow and outcomes of participants though the trial for both clinics combined. Online supplementary web figures and tables describe each clinic separately. We have highlighted below where important differences exist between sites.

Uptake of the study in clinic A was 126/191 (66.0\%), refusers $36 / 191$ (18.8\%). In clinic B 128 of 260 (48.5\%) approached declined to participate (see web table).

Index patients reported 1.4 partners per index for clinic $\mathrm{A}$ and 1.3 partners per index for clinic B of whom 10 (3\%) were uncontactable.

Overall index patients selected APT options for 179/296 (61\%) of their contactable sex partners. However, index patient choice of PN method varied between the clinics (web table). In clinic A index patients selected routine PN for 102/171 (60\%) of their partners with the remainder equally split between APTHotline and APTPharmacy. However, in clinic B, APTHotline was chosen for 100/125 (80\%) of partners.

There were no significant associations between choice of PN option and gender, age, ethnicity, sexual behaviour, index infection, relationship type or duration in either clinic. In clinic A, $25 \%(9 / 36)$ of all indexes who reported more than one

Table 1 Summary of process evaluation

\begin{tabular}{|c|c|c|c|c|c|c|c|}
\hline & \multirow[b]{3}{*}{ Method of evaluation } & \multicolumn{6}{|c|}{ Partner notification (PN) method } \\
\hline & & \multicolumn{2}{|l|}{ Routine } & \multicolumn{2}{|c|}{ APTHotline } & \multicolumn{2}{|c|}{ APTPharmacy } \\
\hline & & Clinic A & Clinic B & Clinic A & Clinic B & Clinic A & Clinic B \\
\hline \multicolumn{8}{|l|}{ Outcome } \\
\hline \multirow[t]{3}{*}{ Patient acceptability } & Index follow-up telephone interview $4-6$ weeks after diagnosis* & $\mathrm{x}$ & $\mathrm{x}$ & $\mathrm{x}$ & $\mathrm{x}$ & $\mathrm{x}$ & $\mathrm{x}$ \\
\hline & Contact follow-up telephone interview $4-6$ weeks after diagnosis $†$ & & & $x$ & $x$ & $x$ & $x$ \\
\hline & Observation and timing of recruitment practice $\neq$ & $\mathrm{x}$ & $\mathrm{x}$ & $x$ & $x$ & $x$ & $x$ \\
\hline \multirow[t]{3}{*}{ Patient feasibility } & Index follow-up telephone interview $4-6$ weeks after diagnosis* & $x$ & $\mathrm{x}$ & $x$ & $\mathrm{x}$ & $x$ & $x$ \\
\hline & Contact follow-up telephone interview $4-6$ weeks after diagnosis $†$ & & & $\mathrm{x}$ & $\mathrm{x}$ & $x$ & $\mathrm{x}$ \\
\hline & 'Mystery shopper' telephone call & & & $\mathrm{x}$ & $\mathrm{x}$ & & \\
\hline Provider acceptability & $\begin{array}{l}\text { Interviews with clinic staff and community pharmacists during and } \\
\text { after trial§ }\end{array}$ & & & $\mathrm{x}$ & $\mathrm{x}$ & $\mathrm{x}$ & $\mathrm{x}$ \\
\hline Provider feasibility & $\begin{array}{l}\text { Interviews with clinic staff and community pharmacists during and } \\
\text { after trial§ }\end{array}$ & & & $\mathrm{x}$ & $x$ & $\mathrm{x}$ & $\mathrm{x}$ \\
\hline
\end{tabular}

\footnotetext{
*Questions included acceptability and feasibility of the PN method used, whether the index would choose that method of PN again, index-reported PN outcomes.

tQuestions included acceptability and feasibility of the PN method used, whether the contact would be happy with that method of PN again, timing of treatment and reasons for this, whether the contact had sought testing for HIV and syphilis after receiving APT and reasons for not doing so.

¥Study researcher observed clinic health advisers as they sought consent from eligible index patients. Health advisers recorded duration of consent process.

§Questions included acceptability and feasibility of the recruitment process, opinions on elements of APT interventions.

APT, Accelerated Partner Therapy.
} 
Index patients recruited between: 05/11/2007 - 01/107/2008

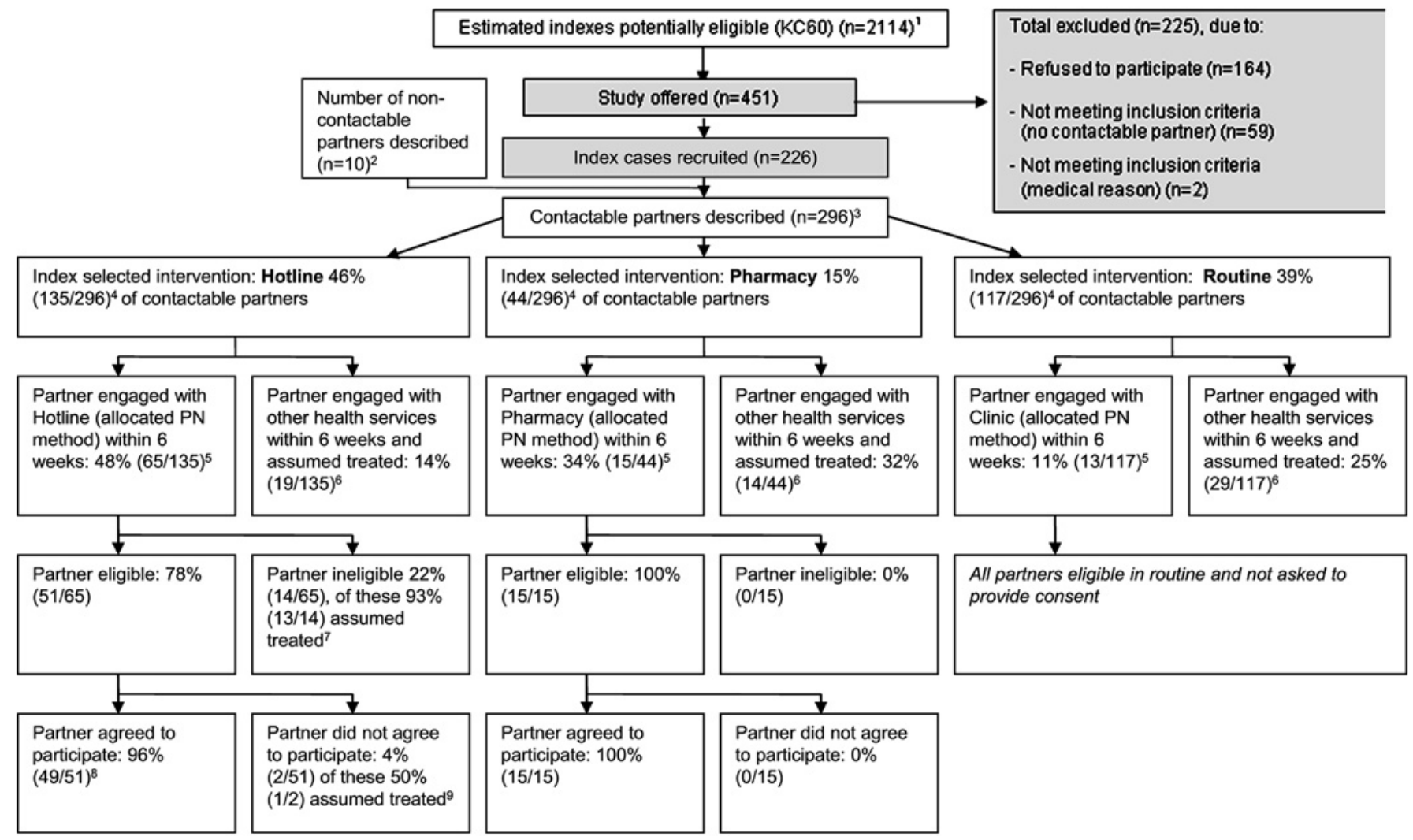

Figure 2 Accelerated Partner Therapy (APT) index patient and sex partner flow diagram, clinics A and B. (1) Number of clinic attenders diagnosed with chlamydia, gonorrhoea or non-gonococcal non-chlamydial urethritis during the study period. Data from KC60 codes. (2) Number of noncontactable partners described by recruited indexes. (3) The total number of partners who were attached to recruited index patients. (4) Total number of partners allocated to each partner notification (PN) method (as selected by the index patient). (5) Number of partners who engaged with the PN method chosen for them by the index within 6 weeks of index diagnosis. No partners engaged after 6 weeks. (6) Number of partners who did not engage with the PN method chosen for them by the index but who did attend other health services within 6 weeks of index diagnosis. No partners engaged after 6 weeks. (7) These partners had symptoms of complicated infection which were identified by the health adviser during the clinical APTHotline assessment. All but one (lost to follow-up) attended clinic without delay and were fully managed according to routine clinic protocol. (8) Two of the 49 partners who engaged with the hotline failed to collect their treatment pack and were assumed not to have been treated. (9) These partners declined to participate in the study.

contactable partner selected different PN options for different partners (clinic B: $6 \%(1 / 18))$.

Thirty-six per cent (42/117) of those contactable sex partners allocated to routine PN were treated (table 2). Treatment was significantly more common among those allocated to APTHotline, $59 \%(80 / 135) \mathrm{p}=0.003$, and those allocated to APTPharmacy, 66\% (29/44) $\mathrm{p}=0.001$ (table 2) compared with routine PN. However, treatment was not always achieved using the APT intervention to which the partners had been allocated. Forty-one per cent (33/80) of the treated partners in the APTHotline group were treated outside the trial for example, GP, other sexual health service, as were $48 \%$ (14/29) of the treated partners in the APTPharmacy group and 29/42 (69\%) routine PN group.

Almost all $(12 / 13,92 \%)$ sex partners undergoing routine PN were tested for $C$ trachomatis, $N$ gonorrhoeae, syphilis and HIV. Under either APT intervention the proportion providing a urine sample for $C$ trachomatis and $N$ gonorrhoeae testing was significantly lower than under routine PN (59\% APTHotline $p=0.03$, $40 \%$ APTPharmacy $\mathrm{p}=0.009$ ), and the uptake of syphilis and HIV testing was extremely poor (4\% APTHotline $\mathrm{p}<0.001,0 \%$ APTPharmacy $\mathrm{p}<0.001$ ), (table 2).

The median time (range) from index diagnosis to partner treatment was $3(0-17)$ days for routine PN and shorter for
APTHotline 1 ( $0-14$ days), $\quad \mathrm{p}=0.05$ and APTPharmacy 2 ( $0-6$ days), $p=0.09$. The median time (range) from index diagnosis to partner treatment where this occurred by the allocated method was $4(0-17)$ days for routine $\mathrm{PN}$ and shorter for APTHotline 1 ( $0-14$ days), $\mathrm{p}=0.03$ and APTPharmacy 1 ( $0-6$ days), $\mathrm{p}=0.11$.

When the statistical tests were repeated in clinic A only, the findings were similar except that the evidence that APTHotline results in faster partner treatment (by allocated method or not) than routine $\mathrm{PN}$ was much weaker, $\mathrm{p}=0.40$.

\section{Process evaluation}

Despite standardised consultation guides for seeking consent, there were differences in approach, perceptions and timing of the process. In clinic $B$, the process took longer than in clinic A and was introduced later in the patient journey through clinic. This may have resulted in loss of potential participants.

The different approaches to consent related to pre-existing PN culture and practices described above. Staff in clinic B may not have perceived equipoise between the three available options in the trial. They felt that their patients strongly preferred the routine approach, and ensured an extremely thorough discussion of all possible advantages and disadvantages while seeking 
Table 2 Partner notification outcomes for sex partners from both clinics according to method selected by index

\begin{tabular}{|c|c|c|c|c|c|c|c|c|}
\hline & \multicolumn{2}{|c|}{$\begin{array}{l}\text { APTHotline } n=135 \\
\text { contactable partners }\end{array}$} & \multicolumn{2}{|c|}{$\begin{array}{l}\text { APTPharmacy } n=44 \\
\text { contactable partners }\end{array}$} & \multicolumn{2}{|c|}{$\begin{array}{l}\text { Routine PN } n=117 \\
\text { contactable partners }\end{array}$} & $\begin{array}{l}\text { Pharmacy } \\
\text { versus routine } \\
\text { p Value }\end{array}$ & $\begin{array}{l}\text { Hotline } \\
\text { versus routine } \\
\text { p Value }\end{array}$ \\
\hline \multicolumn{9}{|l|}{ Primary outcome: } \\
\hline Partners assumed treated by allocated method & $47 / 135$ & 35 (26 to 45$)$ & $15 / 44$ & $34(22$ to 49$)$ & $13 / 117$ & $11(6$ to 19$)$ & 0.001 & $<0.001$ \\
\hline Partners assumed treated by any method & $80 / 135$ & $59(49$ to 69$)$ & $29 / 44$ & 66 (52 to 78$)$ & $42 / 117$ & 36 (26 to 47$)$ & 0.001 & 0.003 \\
\hline \multicolumn{9}{|l|}{ Method of ascertainment of primary outcome: } \\
\hline \multicolumn{9}{|l|}{ Secondary outcomes: } \\
\hline Partner provided sample for STI testing & $29 / 49$ & 59 (45 to 72$)$ & $6 / 15$ & 40 (19 to 66$)$ & $12 / 13$ & $92(60$ to 99$)$ & 0.009 & 0.030 \\
\hline Sample tested & $25 / 49$ & 51 (38 to 64$)$ & $5 / 15$ & $33(14$ to 60$)$ & $12 / 13$ & $92(60$ to 99$)$ & 0.004 & 0.010 \\
\hline Sample positive & $7 / 25 \dagger$ & 28 (14 to 49$)$ & $1 / 5 \ddagger$ & 20 (3 to 71$)$ & \multicolumn{2}{|c|}{ N/A no consent } & $\mathrm{N} / \mathrm{A}$ & $\mathrm{N} / \mathrm{A}$ \\
\hline Partner attended clinic for HIV/syphilis testing & $2 / 49$ & $4(0.5$ to 14$)$ & $0 / 15$ & 0 (0 to 22$)$ & $12 / 13 \S$ & 92 (60 to 99$)$ & $<0.001$ & $<0.001$ \\
\hline
\end{tabular}

*Index patient reported outcomes for partners who engaged with other health services and so were managed outside of trial.

†Seven partners tested positive for $C$ trachomatis.

$\neq$ One partner tested positive for $C$ trachomatis.

§Two partners declined an HIV test but were tested for syphilis.

Samples were tested for the relevant microbiological and virological pathogens according to each clinic's standard testing protocols, which were compliant with current British Association for Sexual Health and HIV National Testing Guidelines.

APT, Accelerated Partner Therapy.

informed consent. This may have deterred patients who preferred routine $\mathrm{PN}$ from participating.

Almost all index patients and contacts found APTHotline and APTPharmacy acceptable and feasible. Some clinic B participants felt that the location of the pharmacies was inconvenient. Sex partners consistently reported that while they understood the need for antibiotic treatment in this context they had not sought HIV and syphilis testing as they did not believe themselves to be at risk.

Mystery shopper calls to the hotlines revealed one instance in each clinic when the telephone was unanswered when the line was advertised as open. Staff felt that the hotline worked optimally when the index patient was able to call his/her partner from clinic and the sex partner chose to undergo immediate telephone consultation with the health adviser.

Pharmacists reported positively on their clinical role. However, at times they experienced difficultly providing adequate trained cover to undertake the consultations. In one pharmacy this necessitated suspending participation during a holiday period.

\section{DISCUSSION}

Overall rates of partner treatment were higher for partners for whom an APT option was chosen by the corresponding index patient, and APT options appear to lead to faster treatment of sex partners as compared with attending clinic for routine PN. Index patients had strong preferences for a particular approach to their partners' PN: 9/34 (26\%) of patients reporting more than one contactable partner chose different PN pathways for them (clinic A). However, this did not appear to reflect partners' own preference, since many actually achieved treatment through a different route. Preferences and recruitment rates varied between sites. This appeared to be related partly to staff satisfaction with their existing routine approach, partly to the approach to consent and possibly, to the characteristics of the local populations. Although $40-60 \%$ of partners in APT groups returned a urine sample for chlamydia and gonorrhoea testing, almost none accessed HIV and syphilis testing.

This is the first evaluation of expedited PN methods that comply with UK regulations. We used recommended methods ${ }^{89}$ for developing the APT interventions and examined both processes and outcomes. Our non-randomised comparative study design allowed us to examine index patients' choices of PN method for their sex partners. The sample size in this preparatory study, however, meant that we had limited statistical power to show associations between choice of PN strategy and partnership type, and that re-infection in the index case was not a feasible outcome. We used a hierarchy of evidence for determining partner treatment and acknowledge that robustness of ascertainment may have differed between groups. However, good concordance between index and partner reports has been demonstrated in the UK. ${ }^{15}$ The only other UK trial of expedited PN was in Scotland. ${ }^{16}$ The uptake of patient-delivered partner therapy, which does not comply with current UK prescribing guidance, was reported to be high. Our low uptake might be surprising as our index patients could choose the most acceptable PN option.

The number of sex partners reported by the index patients was somewhat lower than recent national data. ${ }^{15}$ This is probably owing to exclusion of those with no contactable partners from the trial. The rapid treatment of partners who used APT options is consistent with American studies of patient-delivered partner therapy, ${ }^{10}{ }^{11}$ and suggests that provision of treatment outside traditional settings may reduce barriers to effective PN. This could help prevent re-infection and onward transmission since individuals at risk of STIs may not change their sexual behaviour in the time between awareness of risk and engagement with health services. ${ }^{17}$

Both APT models provide an acceptable point of entry to PN for many index patients and their partners, with potential for high overall partner treatment rates. However, many partners actually achieved their treatment through an alternative pathway and the place of APT as part of a menu of partner notification pathways needs further exploration.

Interventions such as APT, which manage people at risk of STI outside traditional settings, might compromise opportunities to test for HIV in those at higher risk. Low uptake of HIV and syphilis testing in the APT groups is concerning. All UK sexual health clinic attenders should be offered a test for HIV, ${ }^{18}$ and local audits suggest that $93 \%$ of new clinic attenders are being tested (personal communication Mr M Symonds, Barts and the London NHS Trust, June 2010). In a preliminary study 


\section{Key messages}

Accelerated Partner Therapy (APT) models described here are acceptable and feasible new methods of partner notification in UK genitourinary medicine clinics.

- The proportion of partners treated was higher for partners for whom APT was chosen but treatment was not always achieved through APT pathways.

- APT appears to facilitate faster partner treatment than routine partner notification.

- APT needs further exploration in a randomised controlled trial since patient preference may explain some of the enhanced outcomes seen with APT.

of sexual health clinic attenders' views on $\mathrm{APT}^{12}$ participants perceived that they would access HIV and syphilis testing after receiving $\mathrm{APT}$, in contrast to findings in this study. Advances in non-invasive, point-of-care testing technologies might facilitate HIV testing in pharmacies at the time of the APT clinical assessment and the role of self-taken tests needs to be explored.

Patient preferences for different PN options for sex partners might explain some of the enhanced outcomes we saw with APT and future studies should use random allocation. Achieving enrolment at the individual level may be challenging and we suggest cluster randomisation in order to normalise the APT options within individual clinics that have been shown to achieve acceptable outcomes. Initial information and, possibly, informed consent could be sought at the time of testing for an STI, rather than at diagnosis, which can be an anxious time both for patients and for health advisers keen to ensure the best possible outcome for their patients.

\section{Author affiliations}

${ }^{1}$ Centre for Infectious Disease: Sexual Health \& HIV, Blizard Institute of Cell and Molecular Science, Barts \& The London School of Medicine \& Dentistry, Barts and the London NHS Trust, Barts Sexual Health Centre, Kenton \& Lucas Wing, St Bartholomew's Hospital, West Smithfield, London, UK

${ }^{2}$ Centre for Infectious Disease: Sexual Health \& HIV, Blizard Institute of Cell and Molecular Science, Barts \& The London School of Medicine \& Dentistry, West Smithfield, London, UK

${ }^{3}$ Division of Primary Care and Public Health, Brighton and Sussex Medical School, Brighton, UK

${ }^{4}$ Research Department of Infection \& Population Health, Centre for Sexual Health \& HIV Research, University College London, London, UK

${ }^{5}$ Institute of Social and Preventive Medicine, University of Bern, Bern, Switzerland

${ }^{6}$ School of Social and Community Medicine, University of Bristol, Bristol, UK

${ }^{7}$ University Hospitals Bristol NHS Foundation Trust, Bristol, UK

${ }^{8}$ Barts \& The London School of Medicine \& Dentistry, London, UK

${ }^{9}$ Health Economics Unit, University of Birmingham, Birmingham, UK

Acknowledgements We are grateful to the Department of Health, who funded this work through the MRC's Sexual Health and HIV Research Strategy Committee. The opinions expressed in this document are those of the research group and not of the Department of Health or the MRC. We thank the patients, all clinic staff and pharmacists for participating and promoting the research. We would particularly like to acknowledge the help of Dr Matthew Golden, University of Washington, and the following people who have all helped with development of the study: Professor Hunter Handsfield, Dr Eva Jungmann, Dr Gary Brook, Dr John Imrie, Ms K Lucas, collaborators: Dr A Robinson, Dr I Simms.

Funding Department of Health via MRC's Sexual Health and HIV Research Strategy Committee.

\section{Competing interests None.}

Ethics approval This study was conducted with the approval of the Norfolk Research Ethics Committee, REC 06/00101/3.

Contributors CSE was PI, led the research and was lead author; LJS was study researcher and led the conduct of the trial. Study design: CSE, JAC, CM, NML, PH AMJ, LJS, TR, AT; statistical analysis: CM, AC, LJ; data interpretation: all authors; write up: all authors.

Provenance and peer review Not commissioned; externally peer reviewed.

\section{REFERENCES}

1. Anderson RM, Garnett GP. Mathematical models of the transmission and control of sexually transmitted diseases. Sex Transm Dis 2000;27:636-43.

2. Catchpole M. Sexually transmitted infections: control strategies. BMJ 2001;322:1135-6.

3. WHO/UNAIDS. Sexually Transmitted Diseases: Policies and Principles for Prevention and Care. Geneva: UNAIDS. UNAIDS Best Practice Collection, 1999:1-47.

4. Golden MR. Expedited partner therapy: moving from research to practice. Sex Transm Dis 2008;35:320-2.

5. Centres for Disease Control \& Prevention, National Centre for HIV, Viral Hepatitis, STD \& TB Prevention. Expedited Partner Therapy in Management of Sexually Transmitted Diseases. 1600 Clifton Rd, Atlanta, GA 30333, USA. 2006. http://www.cdc.gov/std/ept (accessed 8 Nov 2010).

6. Trelle S, Shang A, Nartey L, et al. Improved effectiveness of partner notification for patients with sexually transmitted infections: systematic review. BMJ 2007; 334:354.

7. Good Practice in Prescribing Medicines. UK: General Medical Council, 2006. http:// www.gmc-uk.org/guidance/current/library/prescriptions_faqs.asp\#5j laccessed 8 Nov 2010).

8. Developing and Evaluating Complex Interventions: New Guidance. London: MRC 2008. http://www.mrc.ac.uk/complexinterventionsguidance (accessed 8 Nov 2010).

9. Craig P, Dieppe $P$, Macintyre $S$, et al. Developing and evaluating complex interventions: the new Medical Research Council guidance. BMJ 2008;337:a1655.

10. Schillinger JA, Kissinger $P$, Calvet $H$, et al. Patient delivered partner treatment with azithromycin to prevent repeat Chlamydia trachomatis infection among women: a randomised controlled trial. Sex Transm Dis 2003;30:49-56.

11. Golden MR, Whittington WL, Handsfield HH, et al. Effect of expedited treatment of sex partners on recurrent or persisting gonorrhoea or Chlamydia infection. $N$ Engl J Med 2005;352:676-85

12. Sutcliffe L, Brook MG, Chapman JL, et al. Is accelerated partner therapy (APT) a feasible and acceptable strategy for rapid partner notification in the UK: a qualitative study of GUM attenders. Int J STD AIDS 2009;20:603-6.

13. Centres for Disease Control \& Prevention, National Centre for HIV/AIDS Viral Hepatitis, STD \& TB Prevention. Program Operations Guidelines for STD Prevention, Surveillance and Data Management. 1600 Clifton Rd, Atlanta, GA 30333 , USA. www.cdc.gov/std/program/surveillance.pdf (accessed 8 Nov 2010).

14. Slater W, Sadler K, Cassell JA, et al. What can be gained from comprehensive disaggregate surveillance. The Avon surveillance for sexually transmitted infections Sex Transm Infect 2007:83/5:411-15.

15. McClean H, Carne C, Bunting P, et al. UK National Audit of chlamydial infection management in sexual health clinics. Case notes audit: information giving, partner notification and follow-up. Int J STD AIDS 2008;19:473-6.

16. Cameron ST, Glasier A, Scot G, et al. Novel interventions to reduce re-infection in women with chlamydia: a randomised controlled trial. Hum Reprod 2009;24:888-95.

17. Mercer $\mathbf{C H}$, Sutcliffe L, Johnson AM, et al. How much do delayed healthcare seeking, delayed care provision, and diversion from primary care contribute to the transmission of STIs? Sex Transm Infect 2007;83:400-5.

18. UK National Guidelines for HIV Testing. http://www.bhiva.org/hivtesting2008.aspx (accessed 8 Nov 2010). 